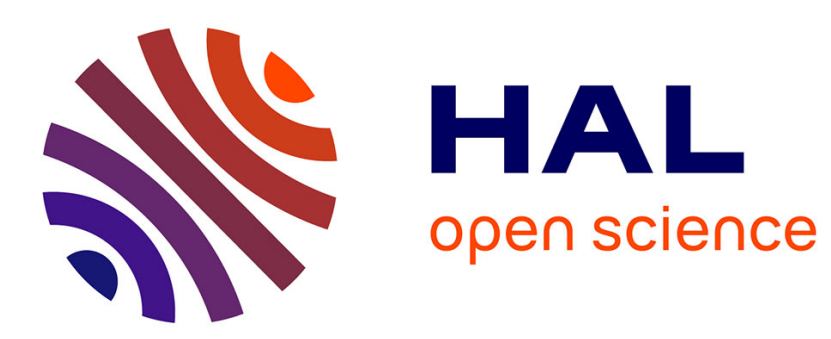

\title{
Universalité des propriétés statiques des polymères en solution semi-diluée
}

\author{
A. Lapp, Cl. Picot, Cl. Strazielle
}

\section{To cite this version:}

A. Lapp, Cl. Picot, Cl. Strazielle. Universalité des propriétés statiques des polymères en solution semi-diluée. Journal de Physique Lettres, 1985, 46 (21), pp.1031-1036. 10.1051/jphyslet:0198500460210103100 . jpa-00232931

\section{HAL Id: jpa-00232931 https://hal.science/jpa-00232931}

Submitted on 1 Jan 1985

HAL is a multi-disciplinary open access archive for the deposit and dissemination of scientific research documents, whether they are published or not. The documents may come from teaching and research institutions in France or abroad, or from public or private research centers.
L'archive ouverte pluridisciplinaire HAL, est destinée au dépôt et à la diffusion de documents scientifiques de niveau recherche, publiés ou non, émanant des établissements d'enseignement et de recherche français ou étrangers, des laboratoires publics ou privés. 
Classification

Physics Abstracts

$61.40 \mathrm{~K}-64.80-82.70$

\title{
Universalité des propriétés statiques des polymères en solution semi-diluée
}

\author{
A. Lapp (*), Cl. Picot and Cl. Strazielle \\ Institut Charles-Sadron (CRM-EAHP), CNRS-ULP Strasbourg, \\ 6, rue Boussingault, 67083 Strasbourg Cedex, France
}

(Reçu le 4 juillet 1985, accepté sous forme définitive le 17 septembre 1985)

\begin{abstract}
Résumé. - La variation de la longueur de corrélation en fonction de la concentration peut être représentée par une courbe universelle indépendante du poids moléculaire, du solvant et de la nature du polymère.
\end{abstract}

Abstract. - The concentration dependence of the correlation length can be represented by a universal plot independent of the molecular weight, the solvent, and the polymer.

\section{Introduction.}

Les propriétés des polymères en solution semi-diluée ont suscité de nombreux travaux aussi bien par diffusion de la lumière (DDL) [1], diffusion des neutrons aux petits angles (DNPA) [2] qu'osmométrie [3]. Ces travaux concernent essentiellement le polystyrène et ont permis de mettre en évidence les exposants caractérisant les variations des paramètres thermodynamiques et les dimensions géométriques statiques avec la concentration. Différentes approches théoriques ont permis de prédire la loi de puissance reliant la longueur de corrélation $\xi$ et la pression osmotique $\pi$ à la concentration. Ainsi M. Daoud et G. Jannink [4] ont établi un diagramme température concentration par une approche tri-critique du point thêta des polymères en solution, M. A. Moore [5] a examiné ces propriétés dans le formalisme de la théorie des champs. Plus récemment M. Muthukamar et S. F. Edwards [6] ont établi des expressions algébriques permettant de relier $\xi$ et $\pi$ à la concentration en tenant compte des effets de volume exclu. $D$. W. Schaefer [7] a montré que les propriétés des solutions de polymères pouvaient être correctement décrites dans un grand domaine de concentration en utilisant une théorie de perturbation dans l'approximation de champ moyen. Une approche différente a été envisagé par T. Ohta et Y. Oono [8] qui, tenant compte de la polydispersité, ont obtenu, par une renormalisation spatiale, des expressions de la variation de la pression osmotique en fonction de la concentration. Cette approche permet d'envisager un traitement universel qui a été utilisé pour décrire par un diagramme unique les propriétés du polystyrène dans des solvants de différentes « qualités » [9].

(*) Détaché du Laboratoire Léon-Brillouin, CEA-CEN Saclay, 91191 Gif sur Yvette. 
Les résultats que nous présentons ont pour objectif de montrer que l'universalité du diagramme peut être étendue à des polymères de natures chimiques diverses (polystyrène, polyméthylméthacrylate, polydiméthylsiloxane), les mesures étant réalisées dans des solvants variés et par des méthodes différentes (DNPA, DDL).

\section{Universalité de la variation de la longueur de corrélation.}

Nous allons établir l'universalité de la variation de la longueur de corrélation tout d'abord en partant des arguments de Daoud et Jannink [4] afin d'établir le type de représentation que nous allons utiliser, puis, nous allons utiliser le formalisme d'Edwards pour établir qu'il s'agit bien d'une représentation universelle.

M. Daoud et G. Jannink ont établi un diagramme reliant le carré moyen de la distance bout à bout $\left\langle\boldsymbol{R}^{2}\right\rangle$ et la longueur de corrélation $\xi$ à la concentration en monomère $c$ et à la température réduite $\tau(\tau=(T-\theta) / \theta)$ pour un polymère ayant $n$ maillons. Dans la région II de ce diagramme correspondant aux solutions semi-diluées nous avons :

$$
\xi \sim c^{-3 / 4} \tau^{-1 / 4}
$$

alors qu'en solution diluée, région $\mathrm{I}$, nous avons

$$
\left\langle R^{2}\right\rangle^{1 / 2} \sim n^{3 / 5} \tau^{1 / 5} .
$$

La concentration de recouvrement étant définie par :

$$
c^{*} \sim n R^{-3} \sim n^{-4 / 5} \tau^{-3 / 5}
$$

nous voyons immédiatement que nous pouvons établir une relation entre $\xi$ et $c$ ne faisant intervenir que des quantités sans dimension :

$$
(\xi / R) \sim\left(c / c^{*}\right)^{-3 / 4} .
$$

L'universalité de la représentation de $(\xi / R)$ en fonction de $\left(c / c^{*}\right)$ peut être établie de manière plus quantitative selon les notations de Muthukumar et Edwards [6] qui tiennent compte du volume exclu $\omega$, paramètre à relier à $\tau$, pour un polymère de $n$ segments ayant chacun une longueur $l$. La longueur de contour est $L=n l$ et la densité en monomère est $\rho(c=\rho=N n / V$ : $N=$ nombre de chaînes).

Par cette approche, il est montré que :

$$
\xi=\left(\frac{9}{16 \pi \alpha_{0}^{1 / 3}}\right)^{3 / 4} \omega^{-1 / 4} l^{-1} \rho^{-3 / 4}
$$

dans un domaine de concentration supérieure à $\rho^{*}\left(\alpha_{0}\right.$ étant un facteur numérique inconnu, voisin de l'unité [6]).

La concentration de recouvrement est définie par :

avec

$$
\rho^{*}=\frac{3}{4 \pi} \frac{n}{R_{G}^{3}}
$$

$$
R_{G}^{2}=\frac{\theta(z)}{6} \times 1,03 \omega^{2 / 5} l^{2 / 5} L^{6 / 5}
$$

où $\theta(z)$ est une fonction variant $\operatorname{avec} z$, le paramètre de volume exclu, qui quantifie l'écart entre le coefficient d'expansion des distances bout à bout $\alpha^{2}$ et celui des rayons de giration $\alpha_{s}^{2}$ [10]

$$
\theta(z)=0,933+0,067\left[\exp -\left(0,853+1,39 z^{2}\right)\right] .
$$


Nous pouvons exprimer $\left(\xi / R_{\mathrm{G}}\right)$ d'une façon analogue à (4), soit :

$$
\begin{aligned}
\left(\xi / R_{\mathrm{G}}\right) & =\left(\frac{3}{4 \alpha_{0}^{1 / 3}}\right)^{3 / 4} \cdot\left(\frac{1,03 \cdot \theta(z)}{6}\right)^{5 / 8}\left(\frac{c}{c^{*}}\right)^{-3 / 4} \\
& \simeq 0,257 \alpha_{0}^{-1 / 4}\left(\frac{c}{c^{*}}\right)^{-3 / 4}
\end{aligned}
$$

en adoptant pour $\theta(z)$ son domaine asymptotique.

Dans cette expression le paramètre de volume exclu et les paramètres caractéristiques du type de polymère utilisé n'apparaissent plus et il est envisageable d'utiliser cette représentation selon un graphe unique pour tous les types de polymères et solvant.

\section{Conditions expérimentales.}

Afin d'explorer un large domaine de concentration nous avons effectué des mesures aussi bien par diffusion de neutrons aux petits angles que par diffusion de la lumière.

La partie neutronique concerne un polydiméthylsiloxane en solution dans le cyclohexane, très bon solvant de ce polymère, et a été effectuée au LLB (Saclay) sur l'appareil de diffusion PACE. La fonction de diffusion permettant d'extraire la longueur de corrélation $\xi$ peut se mettre sous la forme [11]

$$
S(q)=A /\left(1+q^{2} \xi^{2}\right)
$$

où $q$ est le vecteur de diffusion. $\left(q=\frac{4 \pi}{\lambda} \sin \frac{\theta}{2}, \lambda:\right.$ longueur d'onde dans le milieu, $\theta:$ angle de diffusion) et $\boldsymbol{A}$ est une constante dépendant de la constante d'appareillage, du contraste et de la concentration en polymère. Cette forme est utilisable dans un domaine défini par les inégalités suivantes [2] :

$$
1 / R<q<1 / \xi .
$$

La valeur minimum de $\xi$ étant la longueur de l'élément statistique $l$. En DNPA, à $7 \AA$, nous avons couvert un domaine de vecteur de diffusion de : $1,1 \times 10^{-2} \AA^{-1}<q<1,1 \times 10^{-1} \AA^{-1}$. Tandis qu'en DDL, sur un appareil FICA 50 , à $5460 \AA$, ce domaine se situe entre $4 \times 10^{-4} \AA^{-1}$ et $3,2 \times 10^{-3} \AA^{-1}$.

Trois types de polymères ont été utilisés, le polydiméthylsiloxane (PDMS) en solution dans le cyclohexane et dans le toluène, le polyméthylméthacrylate (PMMA) dans le dioxane et le polystyrène (PS) dans le tétrahydrofuranne. Les caractéristiques de ces polymères sont résumées dans le tableau I et les résultats exploités dans le domaine défini par l'inégalité précédente.

Tableau I. - Masse moyenne en poids $M_{\mathrm{w}}$, rayon de giration $\left\langle R_{\mathrm{G}}^{2}\right\rangle_{z}^{1 / 2}$ et concentration de recouvrement $c^{*}$ des polymères.

[Weight average molecular weight $M_{\mathrm{w}}$, radius of gyration $\left\langle R_{\mathrm{G}}^{2}\right\rangle_{z}^{1 / 2}$ and overlap concentration $c^{*}$ for the different polymers.]

\begin{tabular}{l|l|c|c|c} 
Polymère & \multicolumn{1}{|c|}{ Solvant } & $M_{\mathrm{w}}$ & $\left\langle R_{\mathrm{G}}^{2}\right\rangle_{z}^{1 / 2} \AA$ & $c^{*} \mathrm{~g} / \mathrm{cm}^{3}$ \\
\hline PDMS & cyclohexane & $4,2 \times 10^{6}$ & 1490 & $5,03 \times 10^{-4}$ \\
PDMS & toluène (Ref. [12]) & $4,2 \times 10^{6}$ & 1280 & $7,94 \times 10^{-4}$ \\
PMMA & dioxane & $2,8 \times 10^{6}$ & 1043 & $9,89 \times 10^{-4}$ \\
PS 7 & CS $_{2}$ (Ref. [2]) & $5,0 \times 10^{5}$ & 360 & $4,25 \times 10^{-3}$ \\
PS 8 & CS $_{2}$ (Ref. [2]) & $1,1 \times 10^{6}$ & 617 & $1,86 \times 10^{-3}$ \\
PS 447 & THF (Ref. [14]) & $2,87 \times 10^{6}$ & 1123 & $8,03 \times 10^{-4}$
\end{tabular}


Afin de pouvoir normer nos résultats à $c^{*}$, nous avons adopté, pour la définir, la relation suivante :

$$
c^{*}=\frac{3 M_{\mathrm{w}}}{4 \pi \mathcal{N}_{\mathrm{A}}\left\langle R_{\mathrm{G}}^{2}\right\rangle_{\mathrm{z}}^{3 / 2}}
$$

qui montre un bon accord avec l'expérience [13].

\section{Résultats et commentaires.}

A partir des courbes de diffusion de DDL et de DNPA nous avons calculé les longueurs de corrélation, suivant la relation (8), dans le domaine linéaire de cette représentation. Nous avons considéré de plus les résultats de la référence [2] adaptés à nos notations (Tableau II).

Tableau II. - Valeurs réduites des longueurs de corrélation et des concentrations.

[Reduced values of the correlation lengths and concentrations.]

\begin{tabular}{|c|c|c|c|c|}
\hline Polymère & Solvant & $\xi / R_{\mathrm{G}} \times 10^{2}$ & $c / c^{*}$ & Technique \\
\hline PDMS & Cyclohexane & $\begin{array}{l}0,557 \\
0,611 \\
1,09 \\
1,61 \\
2,75 \\
6,20\end{array}$ & $\begin{array}{r}334,0 \\
264,4 \\
166,8 \\
85,9 \\
33,4 \\
15,8\end{array}$ & DNPA \\
\hline PDMS & Toluène & $\begin{array}{l}17,6 \\
27,6 \\
40,3 \\
45,6 \\
46,4 \\
49,1 \\
52,5 \\
55,8\end{array}$ & $\begin{array}{l}4,05 \\
2,00 \\
1,10 \\
0,88 \\
0,54 \\
0,41 \\
0,27 \\
0,14\end{array}$ & DDL \\
\hline PMMA & Dioxane & $\begin{array}{l}13,9 \\
22,8 \\
31,7 \\
32,1 \\
35,8 \\
37,1 \\
47,9\end{array}$ & $\begin{array}{l}4,38 \\
1,99 \\
1,06 \\
1,04 \\
0,78 \\
0,64 \\
0,26\end{array}$ & DDL \\
\hline $\begin{array}{l}\text { PS } 7 \\
\text { PS } 7 \\
\text { PS } 8 \\
\text { PS } 7\end{array}$ & $\mathrm{CS}_{2}$ & $\begin{array}{l}2,92 \\
5,08 \\
7,13 \\
8,28\end{array}$ & $\begin{array}{r}35,3 \\
17,7 \\
13,5 \\
9,4\end{array}$ & DNPA \\
\hline PS 447 & THF & $\begin{array}{l}12,9 \\
15,2 \\
22,3 \\
26,5 \\
33,3 \\
40,4 \\
51,7 \\
53,1\end{array}$ & $\begin{array}{l}5,42 \\
4,07 \\
2,22 \\
1,67 \\
1,11 \\
0,55 \\
0,20 \\
0,15\end{array}$ & DDL \\
\hline
\end{tabular}


L'ensemble des résultats est représenté sur la figure 1 ; on peut constater que dans le domaine de concentration supérieure à $c^{*}$ tous ces points sont regroupés sur une courbe unique qui a pour équation :

$$
\xi / R_{\mathrm{G}}=0,43 \pm 0,015\left(\frac{c}{c^{*}}\right)^{-0,74 \pm 0,01}
$$

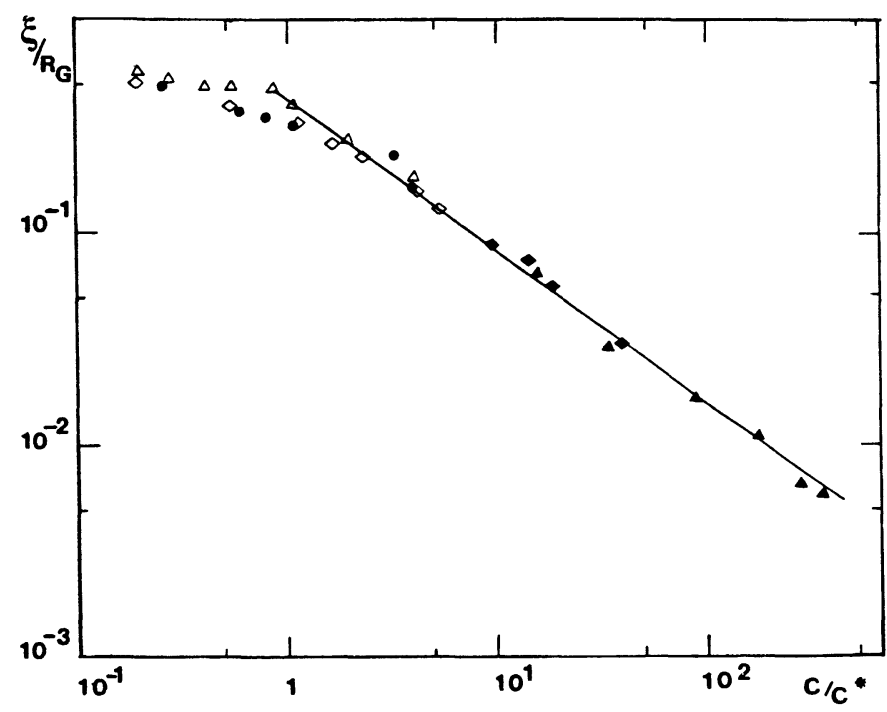

Fig. 1. - Variation de $\log \left(\xi / R_{\mathrm{G}}\right)$ en fonction de $\log \left(c / c^{*}\right)$ pour différents polymères. (A) PDMS : cyclo-

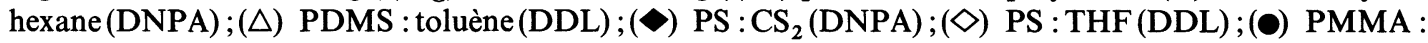
dioxane (DDL).

[Variation of $\log \left(\xi / R_{\mathrm{G}}\right)$ versus $\log \left(c / c^{*}\right)$ for different polymers.]

Les résultats montrent donc de manière précise que la variation de la quantité $\xi / R_{\mathrm{G}}$ est universelle en fonction de $c / c^{*}$ quelles que soient les masses moléculaires, la nature chimique du polymère et du solvant.

Nos résultats font apparaître un exposant de loi de puissance $m$ de $-0,74$. Les théories de lois d'échelle et de renormalisation relient $m$ à l'exposant critique $v$ par $m=-v / 3 v-1$.

En bon solvant, les théories de champ moyen prévoient $v=0,6[15,16]$ tandis que selon la théorie de renormalisation [17] $v=0,588$ ce qui conduit respectivement à $m=-0,75$ et $-0,77$. Nos observations ne permettent pas, compte tenu de la précision expérimentale, de confirmer l'une ou l'autre de ces valeurs.

En ce qui concerne le préfacteur de la loi de comportement, nos expériences conduisent à une valeur de 0,43 qui indique, selon la relation $\left(7^{\prime}\right)$ que le paramètre $\alpha_{0}$, semble différer de l'unité tel que le propose Edwards $\left(\alpha_{0, \exp }=0,126\right)$. Une détermination théorique de cette quantité universelle quel que soit le couple polymère-solvant semble donc nécessaire. 


\section{Conclusion.}

Nous avons montré que la longueur de corrélation réduite $\xi / R$ est une fonction universelle du degré de recouvrement des chaînes soit :

$$
\xi / R_{\mathrm{G}}=0,43\left(c / c^{*}\right)^{-0,74}
$$

et cela quel que soit le couple bon solvant-polymère, pour $c>c^{*}$.

Une remarque s'impose quant au choix que nous avons fait pour la concentration de recouvrement, définie par la relation (9).

Cette valeur caractéristique est justifiée par la figure 1 qui présente un changement de régime à $c / c^{*}=1$. Ce changement de régime se manifeste dans une forme très accentuée dans le cas du PDMS, ce qui a déjà été observé pour des mesures de longueur de corrélation dynamique réalisées par Munch [13].

La représentation universelle adoptée au cours de cette étude ne fait pas apparaître une influence marquée de la polydispersité des échantillons étudiés.

\section{Remerciements.}

Nous remercions G. Jannink et J. des Cloizeaux pour leurs suggestions et J. P. Cotton pour ses conseils au cours des expériences de diffusion de neutrons.

\section{Bibliographie}

[1] Benoît, H., Picot, Cl., Pure Appl. Chem. 12 (1966) 545.

[2] Daoud, M., Cotton, J. P., Farnoux, B., Jannink, G., Sarma, G., Benoît, H., Duplessix, R., Picot, Cl. and DE GENNES, P. G., Macromolecules 8 (1976) 804.

[3] Noda, I., Higo, Y., Ueno, N., Fujimoto, T., Macromolecules 7 (1984) 1055.

[4] Daoud, M., Jannink, G., J. Physique 37 (1976) 973.

[5] Moore, M. A., J. Physique 38 (1977) 265.

[6] Muthukumar, M., Edwards, S. F., J. Chem. Phys. 76 (1982) 2720.

[7] Schaefer, D. W., Polymer 25 (1984) 387.

[8] Ohta, T., Oono, Y., Phys. Lett. A 89 (1982) 460.

[9] Wiltzius, P., Haller, H. R., Cannel, D. S., Schaefer, D. W., Phys. Rev. Lett. 51 (1983) 1183.

[10] Domb, C., Barrett, A. J., Polymer 17 (1976) 179.

[11] Edwards, S. F., Proc. Phys. Soc. 88 (1966) 265.

[12] Lapp, A., Herz, J., Strazielle, C., Makromol. Chemie, sous presse.

[13] Munch, J. P., Lemaréchal, P., Candau, S., J. Physique 38 (1977) 1499.

[14] Résultats communiqués par Mlle L. Ould Kaddour.

[15] Flory, P. J., J. Chem. Phys. 17 (1949) 303.

[16] Edwards, S. F., Proc. Phys. Soc. 85 (1965) 613.

[17] Le Guillou, J. C., Zinn-Justin, Phys. Rev. Lett. 39 (1977) 35. 